\title{
A Langevin Model for Fluctuating Contact Angle Behaviour Parametrised using Molecular Dynamics
}

\begin{abstract}
E. R. Smith ${ }^{* a}$, E. A. Müller ${ }^{a}$, R. V. Craster $^{b}$ and O. K. Matar ${ }^{a}$
Molecular dynamics simulations are employed to develop a theoretical model to predict the fluidsolid contact angle as a function of wall-sliding speed incorporating thermal fluctuations. A liquid bridge between counter-sliding walls is studied, with liquid-vapour interface-tracking, to explore the impact of wall-sliding speed on contact angle. The behaviour of the macroscopic contact angle varies linearly over a range of capillary numbers beyond which the liquid bridge pinches off, a behaviour supported by experimental results. Nonetheless, the liquid bridge provides an ideal test case to study molecular scale thermal fluctuations, which are shown to be well described by Gaussian distributions. A Langevin model for contact angle is parametrised to incorporate the mean, fluctuation and auto-correlations over a range of sliding speeds and temperatures. The resulting equations can be used as a proxy for the fully-detailed molecular dynamics simulation allowing them to be integrated within a continuum-scale solver.
\end{abstract}

\section{Introduction}

The default modelling tools for engineering fluid dynamics assume that the continuum hypothesis is valid. For the droplet on a surface, these continuum-scale models require some boundary conditions to be assumed between solid-liquid, liquid-vapour and vapour-solid interfaces. The meeting of these three interfaces at the moving contact line represents the greatest uncertainty in choice of boundary condition. Worse still, the common assumption of no-slip results in a non-integrable stress singularity at the contact line ${ }^{1}$. This paradox can be avoided in a number of ways, including the assumption of a precursor film, some form of slip, a diffuse interface or evaporation and condensation ${ }^{2}$. The complexity of the contact line has resulted in a number of models for the dynamics being applied at the continuum scale, such as hydrodynamic models including Tanner's law ${ }^{3}$, Cox's law ${ }^{4}$, kinetic models such as molecular kinetic theory (MKT) ${ }^{5}$ and kinematic models ${ }^{6}$.

In a recent review, Snoeijer and Andreotti ${ }^{7}$ suggested that combining hydrodynamic and thermally-activated regimes using the full fluctuating hydrodynamics equation ${ }^{8}$ may be the solution to contact line modelling. This would require computational fluid dynamics (CFD) solvers to incorporate stochastic terms, an uncommon step in CFD practice. As most contact line models used in industrial and academic software are equations linking the macroscopic contact angle $\theta$ and the contact line sliding velocity $U$, a relationship of this form would be of far more immediate use. In the spirit of fluctuating hydrodynamics, a solution to combine

\footnotetext{
a Department of Chemical Engineering

${ }^{b}$ Department of Mathematics

Imperial College London, South Kensington Campus, London SW7 2AZ, UK

* E-mail: edward.smith05@imperial.ac.uk.
}

both hydrodynamic and thermal effects is proposed here using a Langevin-style equation. Previous attempts to develop Langevin models for the contact line ${ }^{9}$ as a series of fluctuating blocks met with limited success ${ }^{10}$ and one possible reason for this failure may be that, as noted by Van Kampen ${ }^{11}$, defining internal noise (i.e., from molecular fluctuations) is not possible with data from a macrosystem. The noise term aims to model microscopic information and this can therefore only be parametrised from data obtained using a full molecular model. It is this observation that motivates the model presented here, with the Langevin equation parametrised to be representative of a full molecular dynamics (MD) simulation.

MD simulations have the potential to explicitly model the diffuse and complex nature of the inner region at the molecular scale. Solving Newton's laws for the N-body system yields stickslip between the solid and liquid as well as a liquid-vapour interfaces with only intermolecular interactions assumed. Although it is possible to model the entire droplet spreading process using $\mathrm{MD}^{12}$, molecular simulations of this type, however, require considerable system sizes 5,12 . More importantly, simulation of molecular droplets are limited, by computational constraints, to nano-meter length and pico-second time scales, neither of which are realistic for most simulations. To avoid these limitations, it is desirable to extract generalised contact line behaviour which can then be incorporated in a continuum-scale model. As we are interested only in the motion of the contact line itself, the droplet is not the simplest case from which to extract the contact angle. The shearing of a liquid bridge between two walls models only the contact line in a reference frame moving with the wall velocity. This simulation geometry has the advantage that a steadystate can be obtained, allowing detailed statistics to be collected. 
The sheared bridge is therefore an ideal test bed to explore the complex dynamics associated with contact line motion.

There are a number of papers which have considered the sheared liquid bridge geometry. In the first such study by Thompson and Robbins ${ }^{13}$, an MD simulation of two-phase Couette flow was used to explore the dependence of the contact angle on the wall speed in a microscopic system. Two immiscible LennardJones fluids are sheared and the behaviour of the dynamic contact line between them is shown to be consistent with Cox's law ${ }^{4}$. In later work by the same group, ${ }^{14}$ a number of other continuum static properties were observed, consistent with the predictions of Laplace's and Young's equations.

The dynamic behaviour of the contact angle was also shown to be strongly dependent on different fluid-wall interactions. The study of Thompson et al. ${ }^{14}$ became a test case for one of the first coupled simulations by Hadjiconstantinou ${ }^{15}$, with an MD model of the contact line as part of a CFD simulation. Instead of explicitly linking continuum and molecular models, Qian et al. ${ }^{16}$ used the setup of Thompson et al. ${ }^{14}$ to derive a generalized Navier boundary condition for use at the continuum scale. This is shown to provide a good approximation for the slip at the wall and was applied in a continuum-scale model. Together with the Cahn-Hilliard interfacial free energy, this continuum model shows very good agreement with the molecular data ${ }^{17}$. Ren and $\mathrm{E}^{18}$ employed the same setup as Thompson et al. ${ }^{14}$ and focused on the validity of the various approximations in the continuum model. It was shown that the assumption of linear transport coefficients such as viscosity is good, only breaking down within 2 - $3 \ell$ from the wall, where $\ell$ is the molecular length scale approximately $3.4 \AA$. The work of Gentner et al. ${ }^{19}$ employs LennardJones molecules in a central liquid region only. Strong elastic bonds are applied at the surface with sixteen atom molecules used to hold the liquid region together and prevent evaporation with Tanner's law and MKT also reproduced by the presented results. Experimental studies of liquid bridges also provide some insight into the general behaviour where the study of Wang and McCarthy ${ }^{20}$ observed pinch off while Nelson et al. ${ }^{21}$ studied the angle sliding rate dependence in the presence of electrowetting.

Here we build on the promising results obtained from the MD simulations of a liquid bridge, which have been used to demonstrate validity of Laplace's and Young's equations, the relationship of Cox, MKT and Tanner's law. Our focus is on a detailed model for the three-phase contact angle itself, using cluster-based interface tracking. Liquid-vapour coexistence is modelled instead of the two immiscible Lennard-Jones fluids used in previous work ${ }^{13,16}$. A careful study of fluctuations of the interface and contact line provides insight into this essential microscopic information. The dependence of the liquid bridge slant on wall-sliding speed is obtained, along with probability density functions (PDF) of fluctuations and autocorrelations in the (slant, advancing and receding) contact angles. Combining the mean, PDF and autocorrelations into a novel stochastic model retains the key elements of molecular simulation but allows them to be directly incorporated into a continuum-scale model.

The rest of the manuscript is arranged as follows: in the methodology section, the MD simulation details and surface- tracking techniques are provided. Next, simulations are presented in the results and discussion section starting, in the first subsection with the key properties of the viscosity and surface tension. The sheared liquid bridge is modelled for a range of wall speeds, and the results compared to existing contact line models in the second subsection. The pinch off of the liquid bridge, and the limits of validity of the MD model compared to experimental observations, are also included in this subsection. The fluctuations in contact angle and autocorrelations are presented in the third subsectionover a range of temperatures. A model for the contact angle is proposed based on Langevin dynamics in the fourth and final subsection of the results and discussion section Finally, the conclusions of the work are highlighted in the last section

\section{Methodology}

Molecular Dynamics (MD) simulation models the movement of discrete molecules interacting and evolving as a function of space and time. The dynamics of the molecules are governed by Newton's laws, with the intermolecular interaction forces, $\boldsymbol{f}_{i j}=-\boldsymbol{\nabla} \phi_{i j}$ obtained from the gradient of the Lennard-Jones potential,

$$
\phi_{i j}=4 \varepsilon\left[\left(\frac{\ell}{r_{i j}}\right)^{12}-\left(\frac{\ell}{r_{i j}}\right)^{6}\right]
$$

All dimensions in this work are reduced with respect to a characteristic length $\ell$ which corresponds to a molecular dimension, a characteristic energy $\varepsilon$ which maps to the well depth of the potential and by fitting the Boltzmann constant, $k_{B}$, to unity. In the Lennard-Jones potential we thus set $\ell=1.0$ and the interaction between all molecules $\varepsilon=1.0$ except wall and liquid where $\varepsilon_{\text {wall }}=0.6$ to adjust the degree of wetting. The potential is a function of the separation distance between molecules $i$ and $j, r_{i j}=r_{i}-r_{j}$. A full parametric study of wall interactions, demonstrating the transition from hydrophobic to hydrophilic, is included in the Appendix. The wall interaction of $\varepsilon_{\text {wall }}=0.6$ is chosen to give an equilibrium angle of approximately $70^{\circ}$. The time-step used is $\Delta t=0.005$. The chosen system size is consistent with literature studies ${ }^{13-16,18}$, although a parametric study of different system sizes was also performed. It was found that the angle variation as a function of wall sliding speed appears to behave in a qualitatively similar manner for larger system sizes. Molecular simulation of system sizes beyond hundreds of nano-meters is computationally prohibitive; however, the existence of similar behaviour in experimental studies ${ }^{21}$ suggest the behaviours of the liquid bridge contact line motion, even in the nano-scale system modelled, is a useful approach to understand the evolution of contact line dynamics in larger macroscopic systems.

The initial system is set up with a domain shape of $L_{x}=$ $79.37, L_{y}=31.75, L_{z}=19.05$ with a face-centred-cubic (FCC) lattice at a density of $\rho=1.0$. The liquid and vapour regions are created by randomly removing molecules from the lattice until the desired densities $\left(\rho_{l}=0.5, \rho_{g}=0.005\right)$ are achieved. Liquid is initialised for $50 \%$ of the domain (excluding tethered regions) and this is allowed to equilibrate with the stationary wall prior to the main run so as to form a liquid bridge. The eventual liquid density is $\rho_{l} \approx 0.82$ while the vapour density is $\rho_{g} \approx 0.005$, values 


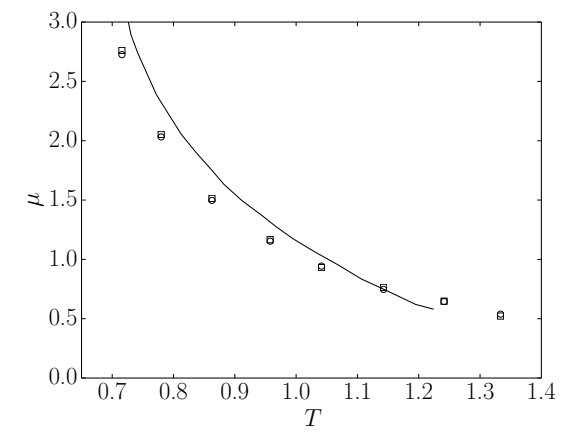

(a)

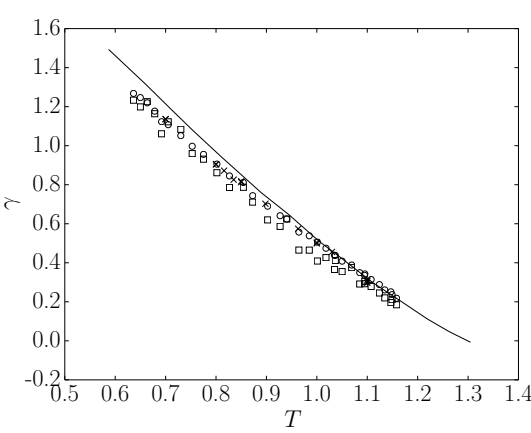

(b)

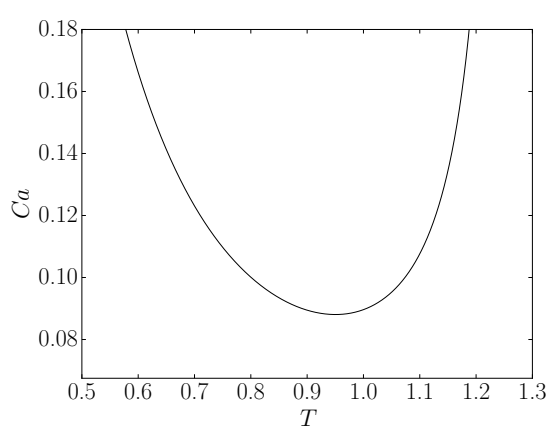

(c)

Fig. 1 Viscosity a) and surface tension b) vs temperature for the Lennard-Jones model of liquid Argon along the triple point, obtained using simulations discussed in the text. The MD results from the volume average stress ${ }^{22,23}(\circ)$ and the method of planes stress ( $\left.\square\right)^{24}$ are compared to digitised experimental results (-) from Refs. ${ }^{25}$ and ${ }^{26}$ in a) and b), respectively. NIST data for equivalent $\operatorname{MD}^{27}(x)$ is also shown in b). In c), we show the variation in the capillary number from Eq. (6) with $U=0.045$ and temperature in the same range as a) and b).

consistent with the expected liquid-gas coexistence at the chosen temperature. Note that the liquid bridge is at higher density and so smaller than the initialised $50 \%$ of the domain. The molecular walls with thickness of 4.0 on top and bottom are modelled by tethering molecules to their equilibrium FCC lattice sites with density $\rho_{s}=1.0$ and spring coefficients ${ }^{28}, k_{2}=0.0, k_{4}=5 \times 10^{3}$ and $k_{6}=5 \times 10^{6}$. A Nosé-Hoover thermostat, setpoint $T_{0}=0.7$ and constant $Q_{\psi}=0.1 N_{\text {thermo }} \Delta t$, is applied to the $N_{\text {thermo }}$ tethered molecules only. Tethered molecules which are adjacent to the liquid, within $1.0 \ell$, are left unthermostatted to allow a temperature profile to develop in the domain and prevent the thermostat from impacting the dynamics of the fluid ${ }^{29}$. The heat bath mass $Q_{\psi}$ is chosen to be a function of time-step and number of thermostatted molecules to ensure the strength of thermostatting is independent of system and time-step size. Only the temperature of the wall is controlled, although as the applied shear is low and there is a recirculation current within the liquid bridge, the temperature in the liquid and solid walls have a difference which is typically less than 0.04 in reduced units, even at the highest wall speeds.

The equations of motion for the wall atoms are given by,

$$
\begin{aligned}
\boldsymbol{v}_{i} & =\frac{\boldsymbol{p}_{i}}{m_{i}}+U_{w} \mathbf{n}_{x}, \\
\dot{\boldsymbol{p}}_{i} & =\boldsymbol{F}_{i}+\boldsymbol{F}_{i_{\mathrm{teth}}}-\psi \boldsymbol{p}_{i}, \\
\boldsymbol{F}_{i_{\mathrm{teth}}} & =\boldsymbol{r}_{i_{0}}\left(4 k_{4} r_{i_{0}}^{2}+6 k_{6} r_{i_{0}}^{4}\right), \\
\dot{\boldsymbol{r}}_{i_{0}} & =U_{w} \mathbf{n}_{x}, \\
\dot{\psi} & =\frac{1}{Q_{\psi}}\left[\sum_{n=1}^{N_{\text {thermo }}} \frac{\boldsymbol{p}_{n} \cdot \boldsymbol{p}_{n}}{m_{n}}-3 T_{0}\right],
\end{aligned}
$$

where $r_{i 0}=r_{i}-r_{0}$ and $r_{0}$ is the tethered molecules equilibrium location which also slides with the prescribed wall speed $U_{w}$ in the $x$ direction as denoted by unit vector $\mathbf{n}_{x}$. The force on a molecule $i$ is the sum of all interactions, $\boldsymbol{F}_{i}=\sum_{j \neq i}^{N} \boldsymbol{f}_{i j}$ and the tethering force $\boldsymbol{F}_{i_{\text {teth }}}$. The molecular velocity in the laboratory reference frame is $\boldsymbol{v}_{i}$ while the momentum in a moving reference frame, the peculiar momentum, is denoted by $\boldsymbol{p}_{i}$. The thermostat variable $\psi$ acts like an integral control on the temperature of the thermostatted wall molecules. All simulations are performed using an in-house MD code which has been fully verified ${ }^{30}$ and used in previous publications ${ }^{31,32}$.

To track the contact line, the location of an intrinsic surface must be defined in the molecular system; the exact location of this purely conceptual surface is arbitrary, and has no effect on the dynamics of the molecular simulation. However, definition of a surface is essential to determine the contact angle observed in the molecular system. A cluster analysis is therefore employed in order to determine molecules which are within the liquid phase. A linklist of molecules is built using the criterion that they are in a cluster when within a length $r_{s} \approx 1.5$ of each other. This approach is computationally simple while being sufficiently robust to detect separate clusters ${ }^{33}$. In order to define the fluid vapour interface, the location of the edge of the liquid cluster must be determined. For a given coordinate direction, a two dimensional grid is defined on the surface of the cluster. By looping through the linked list of molecules in the cluster, the outermost molecule in a given cell is identified and stored. This molecule per cell defines the outer edge of the surface and all molecules within a distance $r_{s}$ of this outer molecule are then identified as surface molecules.

Following the cluster analysis procedure, the bridge surface is then approximated by different functional fits, a one-dimensional linear fit and a cubic polynomial function using the LevenbergMarquardt algorithm from MINPACK. The choice of $r_{s} \approx 1.5$ is a result of a parametric study, balancing good cluster recognition, robust surface definition and efficiency. As there is only one single cluster of interest, the liquid bridge, the choice of $r_{s} \approx 1.5$ was not found to have a noticeable impact on measured angles. This is because angles are defined using a fit to all molecules on the interface and individual molecules entering and leaving do not effect this strongly. During the initialisation of the simulation when there are a number of separate clusters which coalesce, the choice of cluster cutoff, however, does impact angle measurements. For this reason, a period of equilibration is allowed until the system is a single large cluster, and coalescence events are negligible.

The surface tension, $\gamma$, is calculated using the relation of Kirk- 
wood and Buff ${ }^{34}$,

$$
\gamma=\int_{-\infty}^{\infty}\left[\Pi_{x x}-\frac{1}{2}\left(\Pi_{y y}+\Pi_{z z}\right)\right] d x,
$$

where $\Pi_{x x}$ denotes the surface-normal pressure with $\Pi_{y y}$ and $\Pi_{z z}$ the two tangential pressures, and the integral over $x$ crossing both surfaces. Obtaining the pressure is not a trivial exercise, not least because there is no unique form of pressure in a molecular simulation $^{35}$. In this work, two forms of pressure are used in the validation of surface tension and viscosity in Fig. 1 of the next section. The volume average (VA) pressure ${ }^{36,37}$ is simply the integral of the Irving and Kirkwood ${ }^{38}$ form over a volume, in this case a cube,

$$
\int_{V} \Pi d V=-\left\langle\sum_{i=1}^{N} \frac{\boldsymbol{p}_{i} \boldsymbol{p}_{i}}{m_{i}} \vartheta_{i}+\frac{1}{2} \sum_{i, j}^{N} \mathbf{f}_{i j} \boldsymbol{r}_{i j} \int_{0}^{1} \vartheta_{s} d s\right\rangle,
$$

where $\vartheta_{i}$ and $\vartheta_{s}$ are a combination of Heaviside functionals which are non-zero when molecule $i$ or a point on the interaction integral over $s$ between molecules $i$ and $j$ are inside the cubic volume, respectively ${ }^{31}$. The angular brackets denote a time average. In order to obtain the correct local pressure tensor in nonequilibrium MD systems, careful localisation is essential ${ }^{24,39}$. In practice, the pressure must be calculated for a grid of cells (or bins) in order to perform the pressure integral of Eq. (3). There are two contributions to the pressure of Eq. (4). The first contribution uses integer division to assign, $\boldsymbol{p}_{i} \boldsymbol{p}_{i} / m_{i}$, to a cell based on the location of molecule $i$. The second contributions is from intermolecular interactions, $\mathbf{f}_{i j} \boldsymbol{r}_{i j}$. A linear interaction is assumed between two molecules, which is then divided into 20 segments so the stress contribution can be assigned in a piecewise manner to the average volumes which the interaction passes through.

The other route to obtaining the pressure is the method of planes (MOP) ${ }^{24}$, originally derived using the Fourier transformed versions of the Irving and Kirkwood ${ }^{38}$ equations. The MOP pressure given in Eq. (5) is simply the force divided by the area and provides three components on a plane. To get nine components of pressure, a molecular form of Gauss theorem ${ }^{31}$ is used to give a localised form of the method of planes pressure on each surface of the volume:

$$
\oint_{S} \boldsymbol{\Pi} \cdot d \mathbf{S}=-\left\langle\sum_{i=1}^{N} \frac{\boldsymbol{p}_{i} \boldsymbol{p}_{i}}{m_{i}} \cdot d \mathbf{S}_{i}+\frac{1}{2} \sum_{i, j}^{N} \mathbf{f}_{i j} \mathbf{n} \cdot d \mathbf{S}_{i j}\right\rangle,
$$

where $d \mathbf{S}_{i}$ denotes a function which selects the $i^{t h}$ molecule, crossing the surface and $d \mathbf{S}_{i j}$ selects interactions between molecules $i$ and $j$ which cross the surface of a volume of interest. In practice, the plane-line intercept for every surface of every cell in the system must be calculated to determine the pressure of Eq. (5). Every time-step, the movement of molecule $i$ is evaluated and any crossings of a surface contribute $\boldsymbol{p}_{i} \boldsymbol{p}_{i} / \boldsymbol{m}_{i}$ to pressure on the cell's surface. Similarly, all intermolecular interactions below $r_{c}$ are tested and contribute $\mathbf{f}_{i j}$ to the pressure if they cross this surface.

\section{Results and Discussion}

The key dimensionless parameter which describes the flow of the liquid bridge is the capillary number $C a=\mu U / \gamma$, the ratio of vis- (a)



Fig. 2 Comparison of the droplet splitting observed upon shearing in the MD channel, $(a)$, to experimental results, $(b)$, taken from Figure 4 of Wang and McCarthy ${ }^{20}$. Reprinted (adapted) with permission from Wang and McCarthy ${ }^{20}$. Copyright (2013) American Chemical Society.)

cous to capillary forces where $U$ is a characteristic velocity, which in this work is the wall sliding speed $U=U_{w}$, and $\mu$ denotes the viscosity. This section starts by exploring the surface tension and viscosity as a function of temperature, compared to liquid Argon experiments, before moving on to the three-phase contact angle as a function of wall speeds. As shown in the next section, a simple Lennard-Jones model reproduces the temperature dependence of viscosity and surface tension seen in experiments.

\subsection{Surface Tension and Viscosity}

In molecular dynamics, the small system sizes result in shear heating and the change in temperature will affect both viscosity and surface tension. To determine the impact of heating on $\mathrm{Ca}$, parametric studies of viscosity and surface tension are first performed for a range of liquid temperatures. The viscosity of the molecular system is verified using a single-phase fluid sheared between two molecular walls with system size $L_{x}=L_{z}=15.9$ and wall normal $L_{y}=31.75$. The thermostatted walls were set to a density of $\rho_{s}=1.0$, target temperature of $T_{0}=0.7$ with two wall-sliding speeds, $U_{w}=0.5, U_{w}=1.0$ tested for the range of liquid densities $\rho_{l}=0.5-0.85$. The viscosity is calculated by dividing the stress measured in the channel by the strain rate ${ }^{40}$. Good agreement is observed with the experimental results for liquid Argon as shown in Fig. 1a.

Next, the surface tension is calculated for a liquid-vapour coexistence with results shown in Fig. 1b. A separate simulation is performed, as in previous work by Thompson and Robbins ${ }^{13}$, initialised with a separate liquid at the centre in $x$ and vapour phase either side simulated with periodic boundaries in all directions. The domain is of size $L_{x}=41.0$ and $L_{y}=L_{z}=13.68$. This models an infinite film of liquid surrounded on either side by vapour and, after an equilibration period, the integral over the interface between the liquid and vapour phases, Eq. (3), is used to obtain the surface tension $\gamma$. The two different measures of stress, Eqs. (4) and (5) are employed and their predictions show close agreement. The findings of Nijmeijer et al. ${ }^{41}$ indicate too 


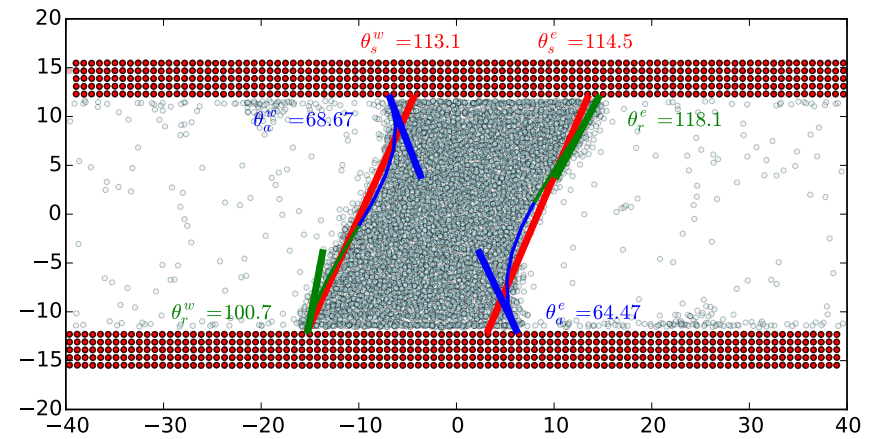

Fig. 3 Molecular dynamics simulation of a liquid bridge sheared between moving walls. The red line corresponding to a linear fit, the blue and green line represent cubic fits of the advancing and receding contact angles, respectively.

small a cutoff can have a pronounced impact on the surface tension. A range of intermolecular potential cutoff distances $r_{c}$ were explored and it was found that $r_{c}=4$ or greater provides closer agreement to experimental data observed in Fig. 1b. MD simulation data from NIST ${ }^{27}$ with the same system setup and cutoff of $r_{c}=4$ are also shown in Fig. 1b and similarly under-predict the experimental data. Improved agreement with experimental data requires a more complex interaction potential and inclusion of three-body interactions ${ }^{42}$. The use of Lennard-Jones with an intermolecular potential cutoff distances $r_{c}=4$ is chosen here as a trade-off between computational efficiency and satisfactory reproduction of experimental surface tension.

By fitting curves to both the viscosity and surface tension data, a temperature dependence of the capillary number, $\mathrm{Ca}$, can be obtained. For viscosity, an inverse power law of the form $\mu(T)=a_{\mu} T^{-3}$ with $a_{\mu}=1.12$ gives an approximate fit. A more complete viscosity-temperature fit requires an expression which is considerably more complex ${ }^{43}$. For surface tension, a good fit is obtained from the Guggenheim scaling approximation $\gamma(T)=a_{\gamma}\left(1-T / T_{c}\right)^{1+b_{\gamma}}$, fitted here to the molecular data instead of the experimental results so $a_{\gamma}=2.9, b_{\gamma}=2 / 9$ and $T_{c}=1.3$. As a result, the capillary number can be seen to be a function of temperature as follows,

$$
C a=\frac{\mu(T) U}{\gamma(T)}=\frac{a_{\mu} U}{a_{\gamma} T^{3}\left(1-T / T_{c}\right)^{1+b_{\gamma}}}
$$

This equation is plotted in Fig. 1c and used throughout the remainder of this work to estimate capillary number dependence on system temperature.

\subsection{Mean Contact Angle}

This section combines two-phase and wall-driven shear flow, to model the liquid bridge using a simple Lennard-Jones fluid. A number of simulations are run with wall speed varied in increments of 0.0025 . At wall velocities above a critical capillary number, the liquid bridge is no longer stable and splits into two droplets. Figure 2 demonstrates that the mechanism seen in the molecular system bears a striking qualitative resemblance to the pinch off observed experimentally ${ }^{20}$. This observation is consis-

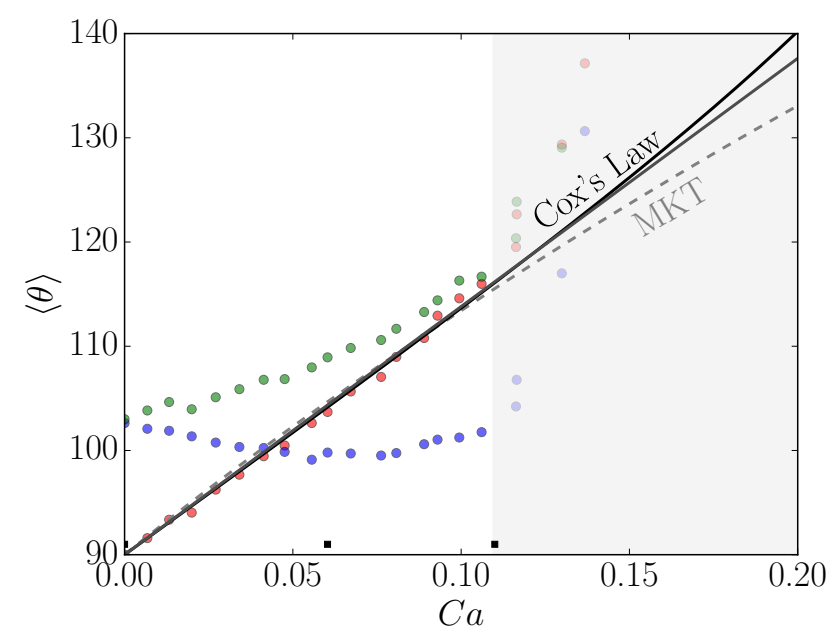

Fig. 4 Variations of the mean angle $\langle\theta\rangle$ with capillary number, $\mathrm{Ca}$, where the liquid bridge slant, $\left\langle\theta_{s}\right\rangle$, advancing, $\left\langle\theta_{a}\right\rangle$, and receding $\left\langle\theta_{r}\right\rangle$ angles are shown in red, blue and green respectively. The lines of fit based on Cox's law and MKT as well as a simple linear fit.

tent with that of Thompson et al. ${ }^{14}$ who also noted that beyond a certain value of wall speed, the liquid bridge breaks up into two parts. The wall sliding speed at liquid bridge pinch off was found in experimental studies to be a function of viscosity ratio and degree of confinement between the walls ${ }^{44}$. The work of Thompson et al. ${ }^{14}$ uses high pressure immiscible liquid phases, resulting in a much wider range of stability than the Lennard-Jones liquidvapour coexistence used in this work.

The interface is tracked using the cluster analysis described in the methodology section. The initial dynamics are characterised by strong transients where several smaller clusters break off and rejoin the main liquid bridge. As the cluster analysis relies on locating extreme molecules, the measured angles are unreliable during this period, and whenever coalescence between smaller clusters occurs. After a time of about 3000 reduced units, the system reaches a non-equilibrium steady state and the mean configuration of the liquid bridge remains constant. At this point, detailed statistics can be collected on the measured contact angles.

A function for the surface is defined using regression, both linear for the overall slant of the bridge (coloured red in Fig. 3) and cubic to estimate the contact angles, both advancing and receding (coloured blue and green in Fig. 3). A cubic fit is employed which, while relatively simple, was judged by eye to sufficiently describe the overall trend in the liquid vapour interface and the near wall contact line as shown in Fig. 3.

The tangent to this cubic function at the wall provides an estimate of the contact angles in the near-wall region. There are two linear 'slant', $\left\{\theta_{s}^{w}, \theta_{s}^{e}\right\}$, two advancing, $\left\{\theta_{a}^{w}, \theta_{a}^{e}\right\}$, and two receding angles, $\left\{\theta_{r}^{w}, \theta_{r}^{e}\right\}$, for the left (west) and right (east) surface, respectively, shown in Fig. 3 as a function of $C a$. The notation $\theta$ is used to define any of the measured slant, advancing or receding angles.

The slant $\left\langle\theta_{s}\right\rangle$ (shown in red in Fig. 4) changes linearly with wall-sliding speed. The advancing angle $\left\langle\theta_{a}\right\rangle$ (shown in blue in 


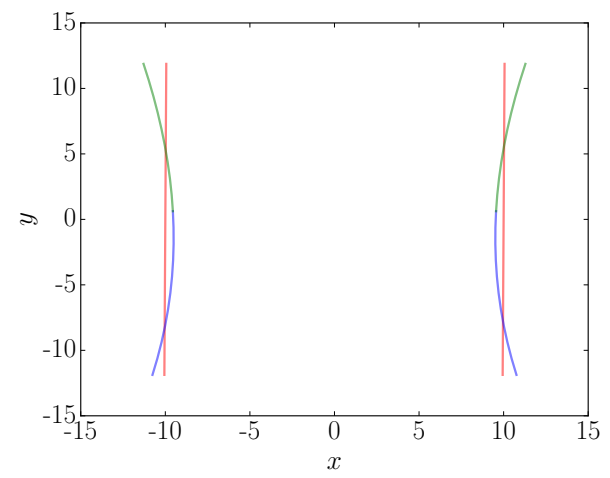

(a)

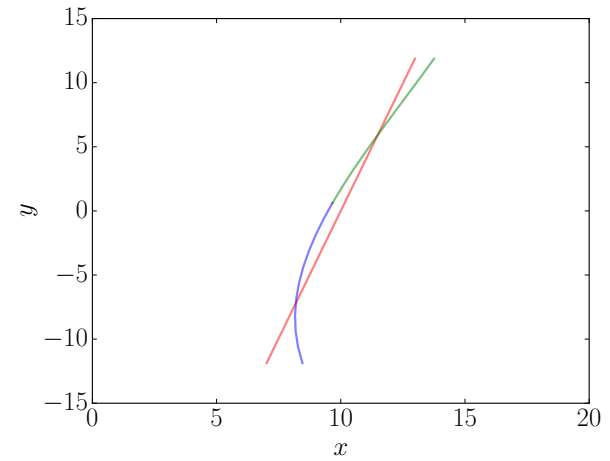

(b)

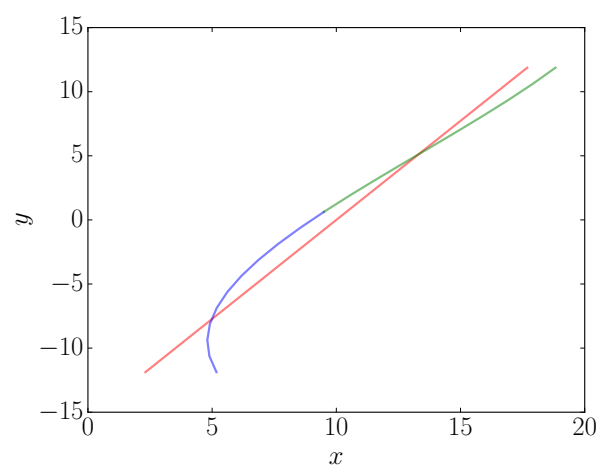

(c)

Fig. 5 Panel a)-c) show the average liquid bridge shape and slant for $C a=0.0,0.0603,0.116$, respectively with surfaces used to define slant, $\left\langle\theta_{s}\right\rangle$, advancing, $\left\langle\theta_{a}\right\rangle$, and receding $\left\langle\theta_{r}\right\rangle$ angles are shown in red, blue and green respectively.

Fig. 4) actually appears to decrease with greater wall speed. This can be attributed to the advancing contact line moving out to stabilise the liquid bridge with increased slant of the bridge in Figs. 5a-5c. The receding angle $\left\langle\theta_{a}\right\rangle$ (shown in green in Fig 4) increases with wall speed less quickly than the slant angle and in a nonlinear manner. For a system with zero wall sliding speed, the advancing and receding angles can be shown to satisfy Young's relation with $\gamma_{L S}-\gamma_{V S}=0.188$ and $\gamma_{L V} \cos \left(\frac{1}{2}\left[\theta_{a}+\left(180-\theta_{r}\right)\right]\right)=0.208$; full details of the calculation process are included in the Appendix. With sliding walls, the slant angle will not satisfy Young's relation, but is nevertheless useful to characterise the average behaviour of the liquid bridge as a function of wall-sliding speed.

There are a number of models for the dynamics of the contact angle at the continuum scale, including Tanner's law ${ }^{3}$, Cox's law ${ }^{4}$ and Molecular kinetic theory (MKT) ${ }^{5}$. Tanner's law is given by,

$$
U=A\left(\langle\theta\rangle-\theta_{e}\right)^{n}
$$

where $A$ and $n$ are arbitrary constants and $\theta_{e}$ is the angle when the contact line is not moving. Note that angular brackets, $\langle\theta\rangle$, are required as the equation governs the behaviour of the average molecular scale contact angle motion. $\mathrm{Cox}^{4}$, assumes an inner length scale with a different behaviour to the outer region and uses matched asymptotics to derive,

$$
C a=\frac{g(\langle\theta\rangle, \lambda)-g\left(\theta_{e}, \lambda\right)}{\ln (L / \ell)-Q_{0} f(\langle\theta\rangle, \lambda)+Q_{i} f\left(\theta_{e}, \lambda\right)},
$$

where $Q_{i}$ and $Q_{0}$ are arbitrary coefficients, $g(\theta, \lambda)=$ $\int_{0}^{\theta} 1 / f\left(\theta^{\prime}, \lambda\right) d \theta^{\prime}$ with function $f(\theta, \lambda)$ a combinations of sines and cosines as given in $\operatorname{Cox}^{4}$ and $\lambda=v_{\text {vapour }} / \nu_{\text {liquid }}$ is the kinematic viscosity ratio between the fluids. Molecular kinetic theory, is based on activated processes inspired by molecular motions but is observed to still be valid at much larger scales ${ }^{2,5}$ :

$$
U=2 \kappa \ell_{j} \sinh \left(\frac{\gamma \ell_{j}^{2}}{2 k_{B} T}\right)\left[\cos \theta_{e}-\cos \langle\theta\rangle\right] \exp \left[\frac{-E}{k_{B} T}\right],
$$

$E$ is the energetic coefficient, $\kappa$ is the frequency of attempted potential jumps and $\ell_{j}$ is the length scale between potential wells due to the movement of fluid over a surface.
The variation of the average bridge slant, $\left\langle\theta_{s}\right\rangle$, with $C a$ is seen to fit very well with the linear part of the MKT and Cox's law theory in Fig. 5. The coefficients used for the fit are consistent with the molecules system, with molecular length scale $\ell=1$, continuum length scale of the MD channel height, $L=23$, arbitrary coefficients $Q_{i}=1$ and $Q_{0}=1$ and the viscosity ratio, $\lambda=0.022$ obtained from NIST data ${ }^{45}$ for Argon in liquid and vapour phases at the appropriate temperature. The MKT fit in Fig. 5 uses $\kappa=1$, and $\ell_{j}=1$, in molecular units, with energetic coefficient, $E=2.0$, temperature, $T=0.7$, based on the thermostat setpoint and most commonly observed temperature in the MD system, with surface tension obtained from the parametrisation of Fig. 1. Finally, Tanner's law is plotted with exponent $n=1$ and $k=0.0042$.

In the literature, the non-linear behaviour of Cox's law ${ }^{13,14}$ and the cubic dependence of Tanner's law ${ }^{19}$ have been observed in a similar molecular geometry. The agreement of molecular scale simulation to macroscopic laws for droplet motions is somewhat surprising. However, average behaviour in molecular simulation often agree very closely with continuum based model, even at very small scales ${ }^{46}$. The slip length for the $\mathrm{LJ}$ potential with the wall-fluid interaction $\varepsilon_{\text {wall }}$ was found to be of order one MD unit in Thompson and Troian ${ }^{47}$ so the âĂIJmolecularâĂİ region of Cox's law may be confined to very near the wall. Only linear agreement between capillary number and angle is observed in this study and this is only valid for slow wall sliding speeds. The nonlinear contact-angle dependence on capillary number, reported in previous work, is seen above $C a=0.11$ in the shaded region of Fig. 5 but the liquid bridge is no longer unconditionally stable at wall-sliding speeds above this. The results presented in Fig 4 of bridge slant with $C a$ suggest that this system is stable only when contact angle is linearly proportional to sliding wall velocity. A similar problem of obtaining a stable liquid-fluid interface is discussed in Ren and $\mathrm{E}^{18}$. These authors attempt to match to MKT, by using high density walls and low wall interactions in order to stabilise the liquid-vapour interface for high wall-sliding speeds. The coefficients required to obtain a good fit, however, are non-physical for the MKT equations, leading Ren and $\mathrm{E}^{18}$ to suggest that the molecular process may be diffusion-dominated, as opposed to the activation-driven mechanism assumed by MKT. 


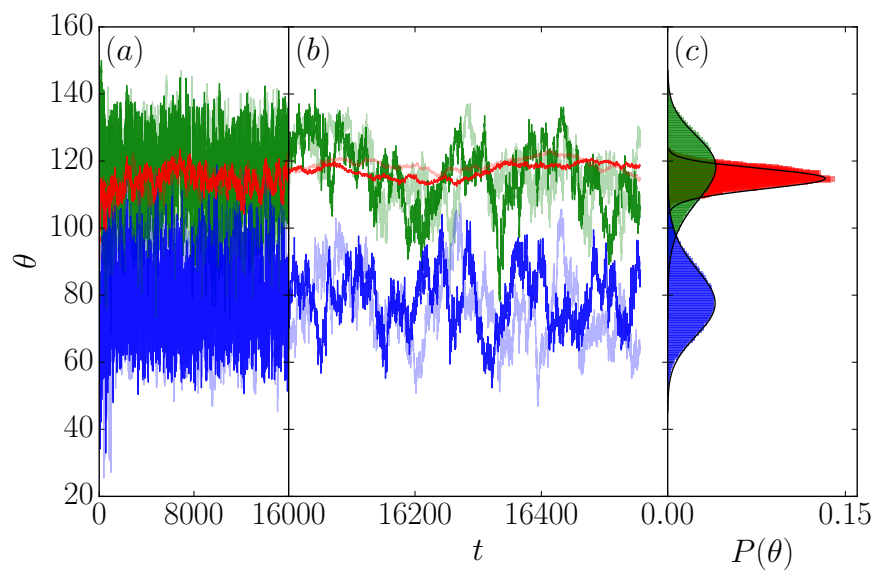

Fig. 6 Time history of contact angle (a) with close up $(b)$ and resulting probability distributions as well as Gaussian fits shown by black line (c) for $C a=0.106$. The red line represents the liquid bridge slant, while the blue and green lines represent the advancing and receding angle, respectively.

The instability of the liquid bridge discussed above and shown in Fig 2 highlights the limitation of the system as a prototypical flow through which one can explore the contact line dynamics. However, the non-equilibrium steady state reached by the liquid bridge at low capillary numbers implies that the long time molecular detail of the contact line dynamics can be studied in detail, paying attention to fluctuations and diffusion processes which are only possible to probe using MD. The next section will therefore measure fluctuations, before proposing a model to build these features into continuum-scale simulation techniques.

\subsection{Fluctuations in Contact Angle}

The temporal evolution of $\theta_{s}, \theta_{r}$ and $\theta_{a}$ is displayed in Fig. 6 with probability density functions (PDF) of the angles shown on the right. The PDFs of fluctuation as a function of time are well fitted by a Gaussian distribution,

$$
f(\theta)=\frac{1}{\sigma \sqrt{2 \pi}} \exp \left[-\frac{[\theta-\langle\theta\rangle]^{2}}{2 \sigma^{2}}\right],
$$

where $\sigma$ is the standard deviation. The black lines in Fig. 6 show Eq. (10) fitted to the raw MD-based PDFs using the LevenbergMarquardt algorithm ${ }^{48}$. There is a much lower standard deviation in contact angle derived from the linear surface fits than the cubic fits used for the advancing and receding contact angles. The use of a linear fit defines a single angle for two surfaces and suppresses individual fluctuations on each surface. The cubic fit is free to model local changes on each surface so is subject to much larger standard deviations. At higher velocities, the PDFs of $\theta_{a}$ and $\theta_{r}$ shows a slight skewed behaviour, potentially due to extreme values in the contact angles at higher speeds.

The standard deviations of contact angle fluctuations, $\sigma_{s}, \sigma_{a}$ and $\sigma_{r}$ are observed to be independent of wall velocity over all cases studied. The wall is an FCC crystal lattice with peaks and troughs, which could be expected to impact the time evolution of $\theta$ in Fig. 6; however, Fourier decomposition of the time se- ries for all measured contact angles shows no clear solid-liquid interaction characteristic of a given wall-sliding speed. The fluctuations in contact angle are therefore assumed to be dominated by thermal motions in the fluid instead of the wall-fluid interactions. This is reasonable as the surface in the molecular system is a perfect lattice and, as a result, does not include the microscopic roughness which would be expected to promote pinning and stick-slip behaviour.

The thermal fluctuations in contact angle, $\theta_{s}, \theta_{a}$ and $\theta_{r}$ are studied for a range of temperatures $0.6 \leq T \leq 1.3$ with a fixed wall-sliding speed of $U=0.025$. The standard deviation of angles is presented in Fig. 7 and fitted to an exponential relationship of the form $\sigma=a_{3} e^{b_{3} T}+c_{3}$. Only results from the range 0.7-1.0 are taken as the study shows freezing below 0.7 and evaporation of the liquid above 1.0. The strong dependence on temperature shown in Fig. 7 supports the conclusion that thermal motions in the fluid are the main contributor to fluctuations observed at the contact line.

The autocorrelations of $\theta_{s}, \theta_{a}$ and $\theta_{r}$ for all temperatures and wall-sliding speeds were also analysed with selected results for $\theta_{s}$ presented in Fig. 8. Surprisingly, there was no clear trend in autocorrelation time as a function of temperature and only a very weak increase in autocorrelation time as a function of wall sliding speed. Higher velocities result in a more persistent measured angle while the slant angle $\theta_{s}$ has a much longer decorrelation time than the advancing and receding angles $\theta_{a}$ and $\theta_{r}$. Autocorrelations are known to require substantial statistics, assuming a decorrelation time of 600 based on Fig. 8, error estimates for autocorrelation in Allen and Tildesley ${ }^{49}$ suggest errors as high as $10 \%$ for the $\mathscr{O}\left(10^{5}\right)$ samples used in this work. It is likely that any trend in $\theta$ autocorrelation against wall speed and temperature, as well as possible departure from exponential behaviour, would require significantly longer runs to identify (e.g., runs of $\mathscr{O}\left(10^{7}\right)$ result in errors of $1 \%$ ). However, the overall trend of exponential decorrelation at short times is apparent in all cases and the fitted exponential lines in Fig. 8 appear to describe the data well.

Characterising the distribution of molecular-level fluctuations as Gaussian allows them to be incorporated into a continuumscale simulation. The introduction of noise into a continuum solver, in the field of fluctuating hydrodynamics, attempts to model the impact of the underlying molecular system. In most cases, the level of noise is negligible in system sizes greater than a few nanometers. However, as the evolution of droplets is heavily dependant on the molecular scale detail near the wall, incorporation of these fluctuations is essential in a continuum solver. Indeed, this idea is the basis for molecular scale models, such as the MKT, used to approximate contact line dynamics in terms of forward or backward jumps. In the next section, a Langevin approach is discussed to combine the Gaussian fluctuations, the exponential decorrelation in contact angle as well as the mean behaviour parametrised in the previous section.

\subsection{A Langevin Model}

We want to define a model for the behaviour of the molecular contact angle observed in the previous two sections. In the proposed 


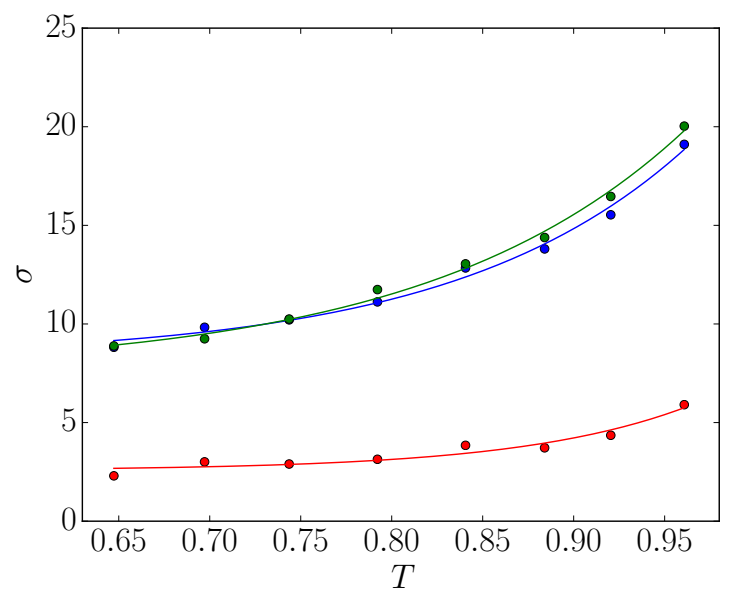

Fig. 7 Standard Deviation in contact angle as a function of temperature for a constant sliding speed of $U=0.025$, with red line for the liquid bridge slant, while the blue and green lines represent the advancing and receding angle, respectively

contact line model, the liquid-vapour interface is assumed to be similar to a Brownian particle: a flexible line, like a strip of metal bent by the drag from the sliding wall and its fluctuations. This conceptual strip is much larger than the atoms which make it up; in the same way Brownian particles are larger than the surrounding molecules. The contact line is deformed by the sliding wall and subject to dissipation and apparent random motions due to thermal collisions with molecules in the wall and fluid. The equation for an angular stochastic harmonic oscillator is postulated to model the liquid bridge contact angle subject to torque applied by the sliding walls,

$$
I \ddot{\theta}(t)+\Gamma \dot{\theta}(t)+k \theta(t)-\xi(t)=\mathscr{T} .
$$

Here, $I$ is the moment of inertia, $\Gamma$ is a damping coefficient, $k$ is the spring constant and $\mathscr{T}$ the applied torque. The stochastic term $\xi(t)$ models the impact of molecular motions on the contact angle. This term satisfies the properties set out in Van Kampen ${ }^{11}$ in that it is stochastic, has zero mean, instantly decorrelates, $\left\langle\xi(t) \xi\left(t^{\prime}\right)\right\rangle=C \delta\left(t-t^{\prime}\right)$, and has a Gaussian distribution. If the inertia of these surface molecules is assumed to be sufficiently small compared to thermal motions, then $I \ddot{\theta}$ is assumed negligible. Taking the time average of Eq. (11), with $\langle\dot{\theta}\rangle \approx 0$ and noting $\langle\xi\rangle=0$ by construction, gives $k\langle\theta\rangle=\mathscr{T}$. The resulting equation, is a Langevin equation for contact angle,

$$
\dot{\theta}+\tilde{k}[\theta-\langle\theta\rangle]-\frac{1}{\Gamma} \xi(t)=0
$$

where $\tilde{k} \equiv k / \Gamma$. As the model for the contact angle is the Langevin equation with a perfectly Gaussian distribution, it is equivalent to the Fokker-Planck equation ${ }^{11}$ and the probability density function (PDF) for steady state angle $\theta$ is,

$$
f(\theta)=\sqrt{\frac{\Gamma k}{C \pi}} \exp \left[-\frac{\Gamma k(\theta-\langle\theta\rangle)^{2}}{C}\right]
$$

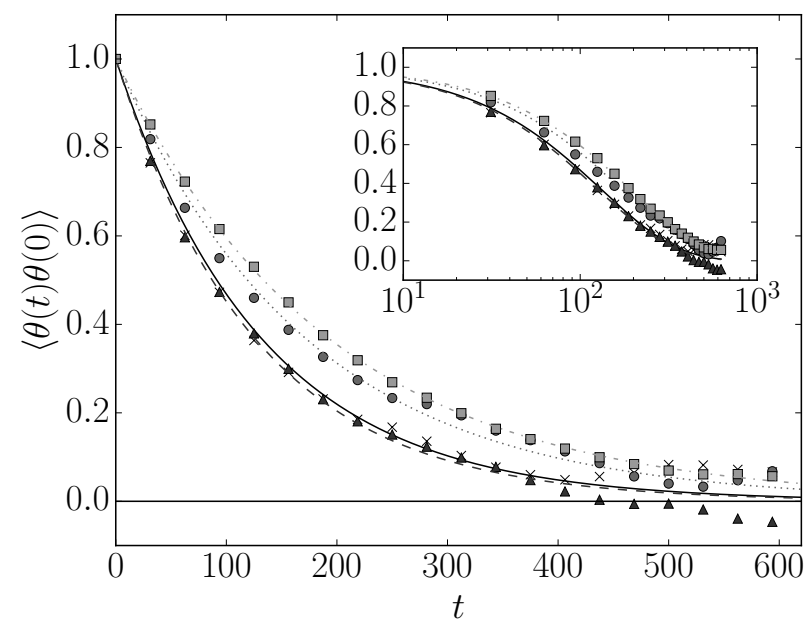

Fig. 8 Autocorrelation of slant angle, $\theta_{s}$, for $\mathrm{Ca}=$ $0.0,0.0067,0.0603,0.0994$ with $\times, \triangle, \circ, \square$ and exponential fit Eq. (14) lines $-,--, \cdot \cdot,-\cdot$, respectively. Insert shows same data with a logarithmic scale on the horizontal axis.

An expression for the autocorrelation of $\theta$ between times $t_{1}$ and $t_{2}$ is then obtained by standard procedures (See e.g., Van Kampen ${ }^{11}$ ):

$$
\left\langle\theta\left(t_{1}\right) \theta\left(t_{2}\right)\right\rangle=\langle\theta\rangle^{2}+\frac{C}{2 k \Gamma} \exp \left(\tilde{k}\left[t_{2}-t_{1}\right]\right) .
$$

With $t_{1}=t_{2}=t$, Eq. (14) is a form of potential energy,

$$
E_{p o t}=\frac{1}{2} k\left\langle\theta^{2}\right\rangle=\frac{1}{2}\left[\frac{C}{2 \Gamma}\right] \approx \frac{k_{B} T}{2} .
$$

Only angle fluctuations around $\langle\theta\rangle$ are assumed to be related to temperature and the $\langle\theta\rangle^{2}$ term is not included. The fluctuations are related to temperature using the equipartition theorem, giving a form of the fluctuation-dissipation theorem for the contact angle, relating dissipation magnitude $\Gamma$ to the magnitude of stochastic fluctuations $C$ and temperature $T$ with an equation of the form $C=2 \Gamma k_{B} T$. The model coefficients, $\langle\theta\rangle, \Gamma, k$ and $C$ can be parametrised using the MD results as follows:

1. The mean of the contact angle $\langle\theta\rangle$ can be expressed using Tanner's law, Cox's law, MKT or another relationship. For liquid bridge slant, $\theta_{s}$, a linear function of velocity could be assumed so $\left\langle\theta_{s}\right\rangle=a_{1} C a+b_{1}$ with constants $a_{1}$ and $b_{1}$ obtained from Fig 5 and $C a$ as a function of temperature from Eq. (6).

2. As the distribution of $\theta$ for a given speed is Gaussian, the standard deviations is relatively invariant to sliding speed but dependant on temperature (see Fig. 7). Therefore, by equating the form of $\sigma=C / 2 \Gamma k$ to the exponential fit to MD standard deviation as a function of temperature $\sigma=a_{3} e^{b_{3} T}+$ $c_{3}$.

3. By fitting the normalised autocorrelation of $\theta$, with mean $\langle\theta\rangle$ removed, to an exponential of the form $\exp \left(-a_{2}\left[t_{2}-t_{1}\right]\right)$, the value for coefficients $\tilde{k}=k / \Gamma=a_{2}$ can be obtained using Eq. (14). As the autocorrelations of Fig. 8 is normalised 

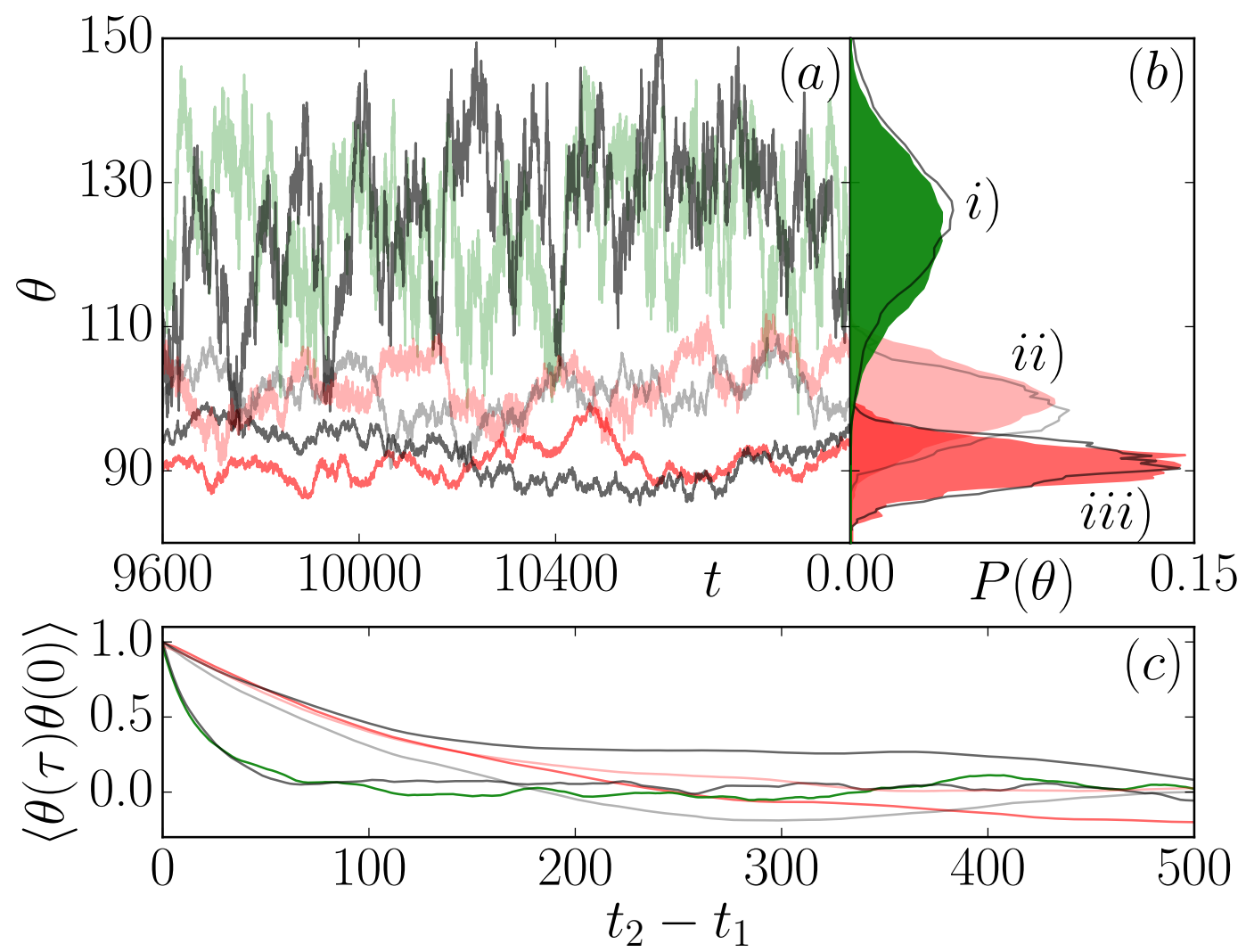

Fig. 9 (a) Time evolution, (b) PDFs and (c) autocorrelations of $i$ ) $\theta_{a}$ for $T=0.7075,\left\langle\theta_{a}\right\rangle=125$ (green); ii) $\theta_{s}$ with $U=0.0200, T=0.912127$ (light red); iii) $U=0.0025, T=0.71388$ (dark red). The Langevin model with tuned coefficients and MD data are shown in black and green/red, respectively. 


\begin{tabular}{|c|c|c|c|}
\hline Coefficient & $\theta_{s}$ & $\theta_{a}$ & $\theta_{r}$ \\
\hline$a_{1}$ & 686.0 & - & - \\
\hline$b_{1}$ & 90.0 & - & - \\
\hline$a_{2}$ & 0.01 to 0.0035 & 0.08 to 0.05 & 0.06 to 0.03 \\
\hline$a_{3}$ & $9.7 \times 10^{-5}$ & $5.68 \times 10^{-3}$ & 0.014 \\
\hline$b_{3}$ & 10.8 & 7.84 & 7.04 \\
\hline$c_{3}$ & 2.57 & 8.25 & 7.58 \\
\hline
\end{tabular}

Table 1 Table of coefficients for three contact angles used in this work

to unity, they are independent of temperature so the single coefficient, $a_{2}$, can be used for the fit.

Therefore, the model coefficients are,

$$
\left\langle\theta_{s}\right\rangle=a_{1} C a+b_{1} ; \quad k=\frac{k_{B} T}{a_{3} e^{b_{3} T}+c_{3}} ; \quad \Gamma=\frac{k}{a_{2}} ; \quad C=2 \Gamma k_{B} T
$$

With these coefficients, the model is tuned to yield the correct mean, time dependant behaviour, distribution and dynamics of the contact angle over a range of wall sliding speeds and temperatures based on the molecular system. The numerical solution of the Langevin equation, Eq. (12), can be written in the form ${ }^{11}$,

$$
\theta^{t+1}=\theta^{t}-\frac{k \Delta t}{\Gamma}\left[\theta^{t}-\langle\theta\rangle\right]+\xi \frac{\sqrt{C \Delta t}}{\Gamma}
$$

By substituting the coefficients from Eq. (16) for the case of slant angle, $\theta=\theta_{s}$, from Table 1 into the Langevin equation,

$$
\theta_{s}^{t+1}=\theta_{s}^{t}-a_{2} \Delta t\left[\theta_{s}^{t}-a_{1} C a-b_{1}\right]+\xi \sqrt{2 a_{2}\left(a_{3} e^{b_{3} T}+c_{3}\right) \Delta t}
$$

which can be compared to MD data, as shown in Fig. 9. The capillary number, $\mathrm{Ca}$, is obtained using average temperatures and wall velocity through Eq. (6). The autocorrelaton coefficients, $a_{2}$, in table 1 are given for fits over the range of minimum to maximum speeds $U=0.0$ to $U=0.425$, with the value, $a_{2}=0.009$ used for $\theta_{s}$ and $a_{2}=0.045$ for $\theta_{a}$ in Fig. 9. Note that the groupings $k / \gamma$ and $C / \gamma^{2}$ in Eq. (17) could have been directly linked to measured relationships from Fig 7 and 8 so that individual values of $k, \gamma$ and $C$ need not be defined. However, as these variables have a physical interpretation in the Langevin equation, Eq. (12), it is useful to obtain them as intermediate variables to gain insight into the dynamics of the molecular system.

The proposed model appears to give good reproduction of the molecular detail with negligible computational effort. Two wall velocities, $U=0.0025$ and $U=0.0200$, with walls thermostatted temperature $T_{0}=0.7$ and $T_{0}=0.9$ respectively are compared to MD results in Fig. 9. The actual system temperatures are slightly different, 0.7075 and 0.912127 , but this is modelled by the Langevin equation with a higher temperature resulting in wider distributions in both MD and Langevin results. The receding angle model at $T_{0}=0.7$ is also presented with a mean value of $\left\langle\theta_{a}\right\rangle=125$ chosen to allow display on the same figure. The shape of the PDFs, autocorrelation and time history of the Langevin model appears to reproduce the MD data well in Fig. 9.

The same time-step and number of iterations were used in the Langevin model, which appears to be statistically under resolved,

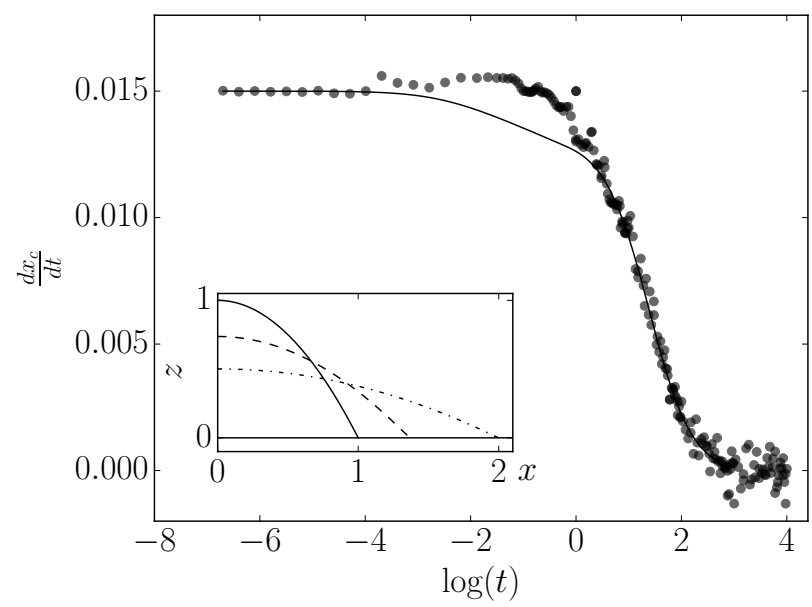

Fig. 10 The contact line velocity as a function of time for a spreading droplet modelled in CFD solver with added MD fluctuations (o) and Tanner's law (-), insert shows droplet interface location at times $t=0.0(-)$, $t=55.0(--)$ and $t=2500.0(\cdot-)$.

resulting in differences of up to $\pm 15 \%$ in model standard deviation. The Langevin model also observes noisy distribution as well as variations in autocorrelation in the same manner as the MD model. These are a direct consequence of the stochastic model employed, recreating the fluctuating nature of molecular dynamics at the contact line. A natural question is how far beyond molecular scales would this be expected to be valid? Blake's ${ }^{5}$ MKT is based on an Eyring style model of a surface as a sinusoid ${ }^{2}$ and despite molecular inspiration is valid to macroscopic scales. For the perfect molecular lattice used in this work the autocorrelations in angle, Fig. 8, show that thermal motion apparently dominates the impact of surface detail. The interaction of the fluid and moving solid lattice would be expected to manifest in the autocorrelation if surface interaction was significant. Blake ${ }^{5}$ observes that system size effects and over simplified surface in molecular models result in non-power law spreading as well as unrealistic (i.e. not experimental) behaviour from MD models. These limitations are with the molecular dynamics used to parameterise the Langevin equation and not the proposed model itself, which is simply a damped torsional spring and additive noise. As this work stems from a molecular scale model, incorporating the molecular thermal motions and other behaviours, it has the potential to provide new insights into contact line modelling. The microscopic origins may also mean this model is valid where other treatments break down.

In any software which uses contact line models, additional stochastic terms can simply be added to model the impact of temperature and molecular-scale effects. A range of other extensions are also possible to address the limitations inherent in the molecular system employed to parametrise this model. For example, the linear relationship between $\langle\theta\rangle$ and wall speed in Eq. (12) could be replaced with Cox's Law, MKT or simply Tanner's law. This can be incorporated into a continuum model in a number of ways. As an example, the Langevin contact line model is implemented in the thin-film finite element CFD solver of Karapetsas et al. ${ }^{50}$, with details of the CFD model given in Appendix A.2. A 
tunable timescale separation is assumed between the molecular and continuum models, chosen here to be $T_{\text {ratio }}=10^{6}$ steps per continuum timestep. The initial contact angle is used as the starting point in Eq. (17), with $T=0.8$ and taking $\theta_{s}$ values from table $1\left(a_{2}=0.01, a_{3}=9.7 \times 10^{5}, b_{3}=10.8, c_{3}=2.57\right)$ and allowed to evolved for time $t=T_{\text {ratio }} \Delta t_{C F D}$ time units. The average of the contact angle from the Langevin evolution over the previous $T_{\text {ratio }}=10^{6}$ timesteps is then used in Tanner's law Eq. (7) at each continuum timestep, with resulting contact line velocity shown in Fig 10. From Fig 10, at short times the contact line motion is dominated by the large difference between $\langle\theta\rangle$ and $\theta_{e}$. At intermediate times, the thermal motions effect the contact line dynamics and it moves more rapidly on average. At longer times, the mean evolution of the contact line is dictated by Tanner's law and follows the the same trends as if there were no fluctuating components. Finally, once the contact angle reaches $\theta_{e}$, the thermal motions will cause it to oscillate about it's equilibrium position. The choice of $T_{\text {ratio }}$ can change the importance of molecular motions on contact line dynamics and incorporate important thermal effects ${ }^{51}$ For coupling to fluctuating hydrodynamics, the mean values and the variance could be exchanged between the molecular and continuum domains, in a similar way to the work of Fabritiis et al. ${ }^{52}$, but with the kinematic closure model for the contact line motion instead of general boundaries.

Modelling of realistic textured surface in large MD systems would be a sensible extension to tune a more detailed Langevin equation. For example, Eq. (12) with applied force in the form of something like $\mathscr{T}=A_{\mathscr{T}} \sin \left(B_{\mathscr{T}} t\right)$ with coefficients $A_{\mathscr{T}}$ and $B_{\mathscr{T}}$ tuned to reproduce surface roughness. It is also possible that more complex physics, such as surfactants or surface coatings, could be course grained in the same manner. The presented model therefore appears to be a very promising for the inclusion of molecule details in larger scale macroscale models.

\section{Conclusion}

The shearing of a molecular liquid bridge includes many complex phenomena, for example temperature-dependent surface tension and viscosity, evaporation, density variation in the fluid, capillary waves on the interface, complex fluid-solid interaction and locally non-linear transport coefficients. The majority of continuum models require a reduced relationship between contact angle and contact line velocity. It is here that the greatest uncertainty lies, and consequently the most benefit to study with molecular scale models.

A systematic MD study of a sheared liquid bridge is presented, including the impact of temperature on viscosity and surface tension. The sheared liquid bridge is only stable in this linear regime with a range of angles spanning approximately $30^{\circ}$; beyond this, pinch off is consistent with experimental observations. The liquid bridge is therefore limited as a method of obtaining mean wall velocity as a function of contact angle, but this non-equilibrium steady state allows unique insight into molecular scale fluctuations at the contact line.

The fluctuations of the contact angle are shown to be well described by a Gaussian distribution in most cases, with some skew at higher speeds. The impact of temperature and sliding speed on these fluctuations is explored. A Langevin model is introduced which includes the impact of molecular features as part of a hydrodynamic system. This is parametrised to include MD values for the mean, standard deviations (fluctuations) and time-dependant behaviour (autocorrelations) of contact angle. The fluctuations at the molecular scale are related to temperature, heat capacity, surface capillary fluctuations as well as dissipation. The impact of these quantities can be incorporated in a continuum solver by including fluctuations from MD. As most continuum models include some form of contact line velocity as a function of angle, this model has a wide range of potential applications.

\section{Acknowledgements}

E. R. Smith is supported under the U.K. Engineering and Physical Sciences Research Council platform Grant EP/L020564/1 and EPSRC MEMPHIS program grant EP/K003976/1. The authors also acknowledge the financial support from EPSRC grant numbers EP/E016340, EP/J014958, EP/L020564.

\section{A Appendix}

\section{A.1 Wall fluid interaction and Equilbirum angle}

The strength of the wall-fluid interaction, $\varepsilon_{\text {wall }}$, can be seen to have a large impact on the measured angles at equilibrium and beyond. This behaviour is parametrised in Figure 11 by measuring the equilibrium contact angle for a range of $\varepsilon_{\text {wall }}$ values. The angle defined by the linear fit is 90 degrees for all values of wall interaction, while the advancing and receding angle vary between 40 and 140 degrees. Note that at extreme values, the cubic fit is poor and the angle is under predicted for the hydrophobic case (should go over 150 and become super-hydrophobic) as well as overpredicted for the hydrophillic case. Also, freezing of the liquid bridge is observed in the strongly hydrophilic case.

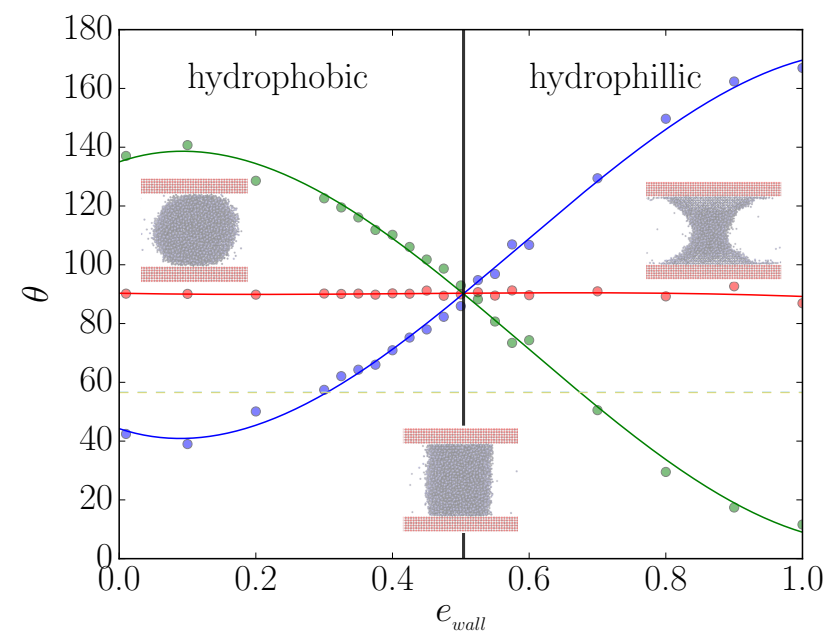

Fig. 11 The change in the three contact angles as a function of wall fluid interaction strength (wetting potential).

The wall fluid potential chosen is $\varepsilon_{w a l l}=0.6$, giving a mildly hydrophilic wall interaction with advancing contact angle around 70 degrees. Other choices of $\varepsilon_{\text {wall }}$ would be equally valid, although the resulting dynamics as a function of sliding appear to 




Fig. 12 Verification of Young's relation, with a snapshot of the MD liquid bridge in the centre, with instantaneous angle, not the average. The corresponding $\Pi_{N}-\Pi_{t}$ pressure differences used in Eq. (3) for surface tension is shown as a function of channel position is shown on the right and bottom of the figure.

follow similar trends.

It can be shown that Young's relationship is valid for the chosen wall interaction of $E_{\text {wall }}=0.6$. The surface tensions and angle of the liquid bridge at equilibrium should satisfy,

$$
\gamma_{L S}-\gamma_{V S}=\gamma_{L V} \cos \left(\frac{1}{2}\left[\theta_{a}+\left(180-\theta_{r}\right)\right]\right)
$$

in an time average sense. This is demonstrated for the liquid bridge by obtaining the surface tension, using the Kirkwood and Buff ${ }^{34}$ definition of Eq. (3), applied in the liquid and gas portions of the domain separately. The liquid-vapour surface tension is $\gamma_{L V}=0.754$, while liquid-solid and solid-vapour are $\gamma_{L S}=0.634$ and $\gamma_{S V}=0.446$ respectively. The stress is calculated at a sufficient distance away from the liquid-vapour interface so only interaction between wall and fluid or wall and gas are considered.

The distribution of the Kirkwood buff integrand is shown in Fig 12. The two sides of Young's relation, Eq. (19), are seen to agree within $10 \%$, which is comparable to the discrepancy of up to $20 \%$ observed in Thompson et al. ${ }^{14}$. This is attributed by Thompson et al. ${ }^{14}$ to residual stresses in the solid and the microscale invalidity of Young's relation ${ }^{53}$.

\section{A.2 Continuum Model Overview}

This section provides a brief overview of the two dimensional CFD solver employed to demonstrate the Langevin contact line model in practice. It is based on the solver of Karapetsas et al. ${ }^{50}$ for the case with no surfactants and the reader is referred to this work for full details. The thin-film form of the non-dimensionalised continuity and momentum equations are assumed,

$$
\frac{\partial u}{\partial x}+\frac{\partial w}{\partial z}=0 ; \quad \frac{\partial P}{\partial x}=\frac{\partial^{2} u}{\partial z^{2}} ; \quad \frac{\partial P}{\partial z}=0
$$

where $z$ is the wall normal direction with velocity $w$ and $u$ is the streamwise velocity in $x$. These equations are solved for a droplet with boundary conditions given by,

$$
\begin{aligned}
u=\beta \frac{\partial u}{\partial z} & \text { at } z=0, \\
\frac{\partial h}{\partial t}+u \frac{\partial h}{\partial x}=w ; \quad P=-\varepsilon^{2} \gamma \frac{\partial^{2} h}{\partial x^{2}} & \text { at } z=h,
\end{aligned}
$$

and initial condition,

$$
h(x, t=0)=1-x^{2} ; x_{c}(t=0)=1 .
$$

The contact line velocity evolves according to Tanner's law, Eq. (7) combined with the molecular Langevin equation. The equations are solved using the finite element method, NewtonRaphson iteration with adaptive timestep $\Delta t_{C F D}$ varying from $10^{-7}$ to $10^{-2}$. The slip coefficient is $\beta=10^{-5}$, the ratio of height to width is $\varepsilon=0.42$, surface tension is $\gamma=1.0$, equilibrium angle $\theta_{e}=28.65$ degrees and Tanner's Law coefficients are $A=0.001$ and $n=1$ for both the case which uses the Langevin equation and without. Three hundred elements were used for both liquid and vapour regions and scaled coordinates $\eta$ are employed using the location of the contact line for both the liquid, $\eta=x / x_{c}$, and vapour, $\eta=2-x / x_{c}$.

\section{References}

1 C. Huh and L. Scriven, Journal of Colloid and Interface Science, 1971, 35, 85 - 101.

2 D. Bonn, J. Eggers, J. Indekeu, J. Meunier and E. Rolley, Rev. Mod. Phys., 2009, 81, 739-805.

3 L. H. Tanner, Journal of Physics D: Applied Physics, 1979, 12, 1473.

4 R. G. Cox, Journal of Fluid Mechanics, 1986, 168, 169-194.

5 T. D. Blake, Journal of Colloid and Interface Science, 2006, 299, 1 - 13.

6 Y. D. Shikhmurzaev, Journal of Fluid Mechanics, 1997, 334, 211-249.

7 J. H. Snoeijer and B. Andreotti, Annu Rev Fluid Mech, 2013, 45, 269-292.

8 E. G. Flekkøy and D. H. Rothman, Phys. Rev. E, 1996, 53, 1622-1643.

9 A. Douglas, C. Pierre, C. Joël and D. Franccois, Journal of Statistical Physics, 1990, 61, 509-532.

10 M. Voué and J. D. Coninck, Acta Materialia, 2000, 48, 4405 4417.

11 N. G. Van Kampen, Stochastic Processes in Physics and Chemistry, 3rd edn., 2011.

12 P. E. Theodorakis, E. A. Muller, R. V. Craster and O. K. Matar, Soft Matter, 2015, 11, 9254-9261.

13 P. A. Thompson and M. O. Robbins, Phys. Rev. Lett., 1989, 63, 766-769.

14 P. A. Thompson, W. B. Brinckerhoff and M. O. Robbins, J. Ad. Sci. Tech., 1993, 7, 535-554.

15 N. G. Hadjiconstantinou, Ph.D. thesis, MIT (U.S.), 1998. 
16 T. Qian, X.-P. Wang and P. Sheng, Phys. Rev. E, 2003, 68, 016306.

17 T. Qian, X. P. Wang and P. Sheng, Commun. Comput. Phys., 2005, 1, 1-52. 36 p.

18 W. Ren and W. E, Physics of Fluids, 2007, 19, 022101.

19 F. Gentner, G. Ogonowski and J. D. Coninck, Langmuir, 2003, 19, 3996-4003.

20 L. Wang and T. J. McCarthy, Langmuir, 2013, 29, 7776-7781.

21 W. C. Nelson, P. Sen and C. Kim, Langmuir, 2011, 27, 1031910326.

22 R. J. Hardy, J. Chem. Phys., 1982, 76, 622.

23 J. F. Lutsko, J. Appl. Phys., 1988, 64, 1152.

24 B. D. Todd, D. J. Evans and P. J. Daivis, Phys. Rev. E, 1995, 52, 1627.

25 W. T. Ashurst and W. G. Hoover, Phys. Rev. Lett., 1973, 31, 206-208.

26 B. Shi, Ph.D. thesis, University of California Los Angeles. USA, 2006.

27 Lennard-Jones fluid simulation website 6. Surface Tension, URL: http: //www.cstl.nist.gov/srs $/ L J_{P} U R E /$ surface $_{t}$ ension.htm, Accessed: 2016-04-06.

28 J. Petravic and P. Harrowell, J. Chem. Phys., 2006, 124, 014103.

29 S. Bernardi, B. D. Todd and D. J. Searles, J. Chem. Phys., 2010, 132, 244706.

30 E. R. Smith, Ph.D. thesis, Imperial College London, 2014.

31 E. R. Smith, D. M. Heyes, D. Dini and T. A. Zaki, Phys. Rev. E., 2012, 85, 056705.

32 E. R. Smith, Phys. Fluids, 2015, 27, 115105.

33 D. C. Rapaport, The Art of Molecular Dynamics Simulation, Cambridge University Press, Cambridge, 2nd edn., 2004.

34 J. G. Kirkwood and F. P. Buff, The Journal of Chemical Physics, 1949, 17, 338-343.

35 P. Schofield and J. R. Henderson, Proc. R. Soc. Lond. A, 1982, 379, 231.

36 C. Hirsch, Numerical Computation of Internal and External
Flows, Elsevier, Oxford, 2nd edn., 2007.

37 J. Cormier, J. M. Rickman and T. J. Delph, J. Appl. Phys., 2001, 89, 99.

38 J. H. Irving and J. G. Kirkwood, J. Chem. Phys., 1950, 18, 817.

39 D. M. Heyes, E. R. Smith, D. Dini and T. A. Zaki, J. Chem. Phys., 2011, 135, 024512.

40 D. J. Evans and G. P. Morriss, Statistical Mechanics of NonEquilibrium Liquids, Australian National University Press, Canberra, 2nd edn., 2007.

41 M. J. P. Nijmeijer, A. F. Bakker, C. Bruin and J. H. Sikkenk, The Journal of Chemical Physics, 1988, 89, 3789-3792.

42 A. Ghoufi, P. Malfreyt and D. J. Tildesley, Chem. Soc. Rev., 2016, 45, 1387-1409.

43 B. A. Younglove and H. J. M. Hanley, Journal of Physical and Chemical Reference Data, 1986, 15, 1323-1337.

44 A. Vananroye, P. V. Puyvelde and P. Moldenaers, Langmuir, 2006, 22, 3972-3974.

45 E. W. Lemmon and S. G. Penoncello, in Advances in Cryogenic Engineering, Springer US, Boston, MA, 1994, ch. The Surface Tension of Air and Air Component Mixtures, pp. 1927-1934.

46 K. P. Travis, B. D. Todd and D. J. Evans, Phys. Rev. E, 1997, 55, 4288-4295.

47 P. A. Thompson and S. M. Troian, Nature, 1997, 389, 360362.

48 J. J. Moré, B. S. Garbow and K. E. Hillstrom, User guide for MINPACK-1, Cm-p00068642 technical report, 1980.

49 M. P. Allen and D. J. Tildesley, Computer Simulation of Liquids, Clarendon Press, Oxford, 1st edn., 1987.

50 G. Karapetsas, R. V. Craster and O. K. Matar, Journal of Fluid Mechanics, 2011, 670, 5-37.

51 G. Grün, K. Mecke and M. Rauscher, Journal of Statistical Physics, 2006, 122, 1261-1291.

52 G. D. Fabritiis, M. Serrano, R. Delgado-Buscalioni and P. Coveney, Phys. Rev. E, 2007, 75, 026307.

53 G. Saville, J. Chem. Soc., Faraday Trans. 2, 1977, 73, 11221132. 